\title{
POEM
}

\section{Gina Betcher}

\author{
Is it a shallow feeling \\ that you and I \\ together share \\ Water \\ ankle high \\ Foot \\ and ankle bare \\ Is it amongst ourselves \\ that you and I \\ together \\ can feel warm \\ United \\ as one \\ Alone together \\ we conform
}

Are we visible injuries that you and I together see As we stand on the shore and share the sea Water ankle high Foot and ankle bare 